\title{
Molecular cloning and characterization of GDP-mannose-3',5'-epimerase from Gracilaria changii
}

\begin{abstract}
GDP-mannose-3 , A A fipimerase (GME) is an enzyme involved in the biosynthesis of GDP-1galactose which is a building unit of agar and cell wall polysaccharides. GME catalyzes the formation of GDP-6-l-galactose and GDP-1-gulose from GDP-mannose. In this study, the gene and transcript encoding GME from the red alga Gracilaria changii (GcGME) were cloned. The structural gene sequence of GcGME is devoid of an intron. The cis-acting regulatory element involved in light response is the most abundant element at the 5 Nijlanking region of GcGME. The open reading frame of GcGME consists of 1,053 nucleotides with 351 amino acids. This cDNA was cloned into pET32a expression vector for recombinant protein production in Escherichia coli. High yield of soluble recombinant GcGME (55 kDa) was expressed upon isopropyl b-d-1-thiogalactopyranoside induction. The enzyme activity of recombinant GcGME was detected using thin layer chromatography and high-performance liquid chromatography. The transcript abundance of GcGME was the highest in G. changii and the lowest in Gracilaria salicornia corresponding to their agar contents. The characterization of GcGME from G. changii is important to facilitate the understanding of its role in agar production of this seaweed.
\end{abstract}

Keyword: GDP-mannose-3N, Alypimerase; Gene expression; Gracilaria changii; Recombinant protein; Rhodophyta 